\title{
Surgical Management of Post-Traumatic Iris Cyst
}

\author{
Swetha Sara Philip*, Deepa Rebecca John, Fini Ninan and Sheeja Susan John
}

Department of Ophthalmology, Christian Medical College, Vellore 632001, Tamil Nadu, India

\begin{abstract}
Purpose: Epithelial cysts of the iris may be primary or secondary. The management of secondary, posttraumatic iris cysts is often challenging. The purpose of this work is to report the successful surgical management of a post-traumatic iris cyst.

Case Report: A 38 year-old man presented with an iris cyst associated with epithelial downgrowth and total cataract, six years after sustaining a penetrating injury to his right eye. The iris cyst was excised in toto with the epithelial downgrowth, and the procedure was combined with cataract extraction and intra ocular lens implantation. The patient had good post-operative visual recovery with minimal inflammatory reaction.

Conclusion: Post-traumatic cysts of the iris can be associated with significant ocular morbidity. Many different modalities of treatment, with varying degrees of success, have been described for the treatment of iris cysts. Excision of the iris cyst in toto is a good surgical management option, which can result in an excellent functional outcome with minimal inflammatory complications.
\end{abstract}

Keywords: Cataract surgery, iris cyst, management, post-traumatic, surgery.

\section{INTRODUCTION}

Epithelial cysts of the iris may be primary or secondary [1]. Secondary iris cysts may occur after intraocular surgery, trauma, inflammation or long-term use of topical medications like miotics [2]. These cysts develop from epithelial cells of the cornea or conjunctiva that have been implanted or transplanted on the iris through the wound [3]. These cells in the anterior chamber then grow into a sheet or form a cyst [4]. Traumatic iris cysts are of two types: the "solid-looking" cyst or pearl cyst has stratified or cubical epithelium lining the cyst wall; the second type is, called the serous cyst, contains straw colored turbid fluid [5].

If the iris cyst is small and asymptomatic, close observation may be sufficient. However, larger iris cysts can grow in size, and cause pupillary block and secondary glaucoma, uveitis, corneal decompensation and eventually, a painful, blind eye [1, 6 , 7]. Many different modalities of treatment, ranging from minimally invasive techniques like aspiration of the cyst and laser therapy, to aggressive surgical procedures like block excision have been described for the management of iris cysts [4]. The purpose of this work is to describe the management of a post-traumatic iris cyst by surgical excision of the cyst in toto, which achieved a good functional outcome with no significant inflammatory complications.

\section{CASE REPORT}

A 38 year-old man presented to the outpatient clinic of our centre with mild pain and redness in the right eye for the

*Address correspondence to this author at the Department of Ophthalmology, Schell Eye Hospital, Arni Road, Christian Medical College, Vellore 632001, Tamil Nadu, India; Tel: +91-416-2281201;

E-mail: anubaby@cmcvellore.ac.in past two days. He also complained of gradually progressive decrease in vision in the right eye for the past one year. $\mathrm{He}$ had sustained a penetrating injury in the right eye six years ago, and had undergone surgical repair of the wound at another centre.

The vision in the right eye was hand movements with accurate projection of rays in all four quadrants. Apart from minimal circumcorneal congestion, there were no signs of ocular inflammation. An irregular scleral scar was noted at the superior limbus at twelve o' clock position, extending about two millimeters posteriorly, with extension into the cornea just within the limbus. A cyst of the iris was seen in the superonasal quadrant, extending from twelve to two o' clock position, associated with epithelial downgrowth and extensive peripheral anterior synechiae from ten to two o' clock position (Fig. 1). A total cataract obscured the view to the fundus. The intraocular pressure was normal and there was no relative afferent pupillary defect. The left eye was normal.

Ultrasound biomicroscopy of the right eye showed a thinwalled iris cyst with internal echolucency. There was no evidence of a foreign body (Fig. 2). B scan ultrasonography of the right eye showed a clear vitreous with no evidence of a foreign body or retinal detachment. The patient underwent surgical excision of the iris cyst with cataract surgery under cover of topical corticosteroids. The cyst wall and peripheral anterior synechiae were separated from the corneal endothelium by gentle viscodissection. Posterior synechiae under the iris cyst were also released by viscodissection, aided by mechanical separation with the cannula used for injection of the viscoelastic agent. After conjunctival peritomy, a limbal incision was made from ten to two o' clock position. The iris cyst was excised in toto, with 


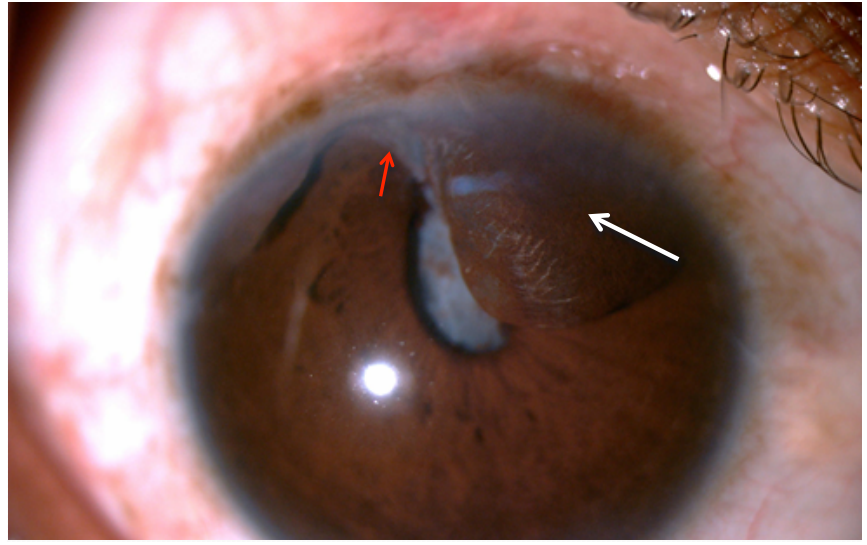

Fig. (1). Slit lamp photograph of the right eye showing the iris cyst (white arrow) and epithelial downgrowth (red arrow).

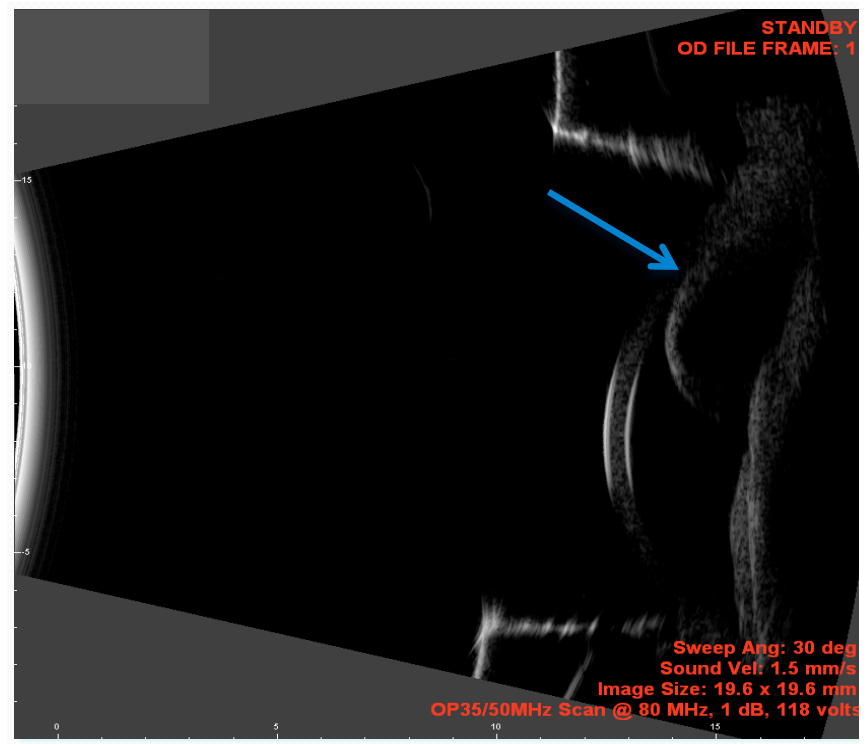

Fig. (2). Ultrasound biomicroscopy showing the echolucent iris cyst (blue arrow).

excision of a one-millimeter margin of surrounding iris tissue. The epithelial downgrowth was also excised, and an extracapsular cataract extraction was performed with implantation of a rigid, single piece intraocular lens in the capsular bag. The limbal incision was sutured with 10-0 nylon sutures. The excised tissue was sent for histopathological examination, and was reported as a benign epithelial cyst of the iris. The post-operative period was uneventful. There was no significant ocular inflammatory reaction, and fundus was normal on dilated ophthalmoscopic examination.

The patient was followed up in our clinic, and the bestcorrected visual acuity in the right eye was 20/20 at the last follow-up visit three months after surgery (Fig. 3).

\section{DISCUSSION}

Secondary iris cysts may occur as a result of penetrating corneal injury or intraocular surgery [3]. The pathogenesis of a secondary iris cyst begins with epithelial cells proliferating in the anterior chamber in the form of a serous cyst, pearl cyst or epithelial downgrowth [8]. These epithelial cells then grow and extend into other ocular structures like the angle and ciliary body $[9,10]$. Small and stable cysts may be closely observed for signs of progression or complications like intraocular inflammation. The larger cysts require surgical management [11].

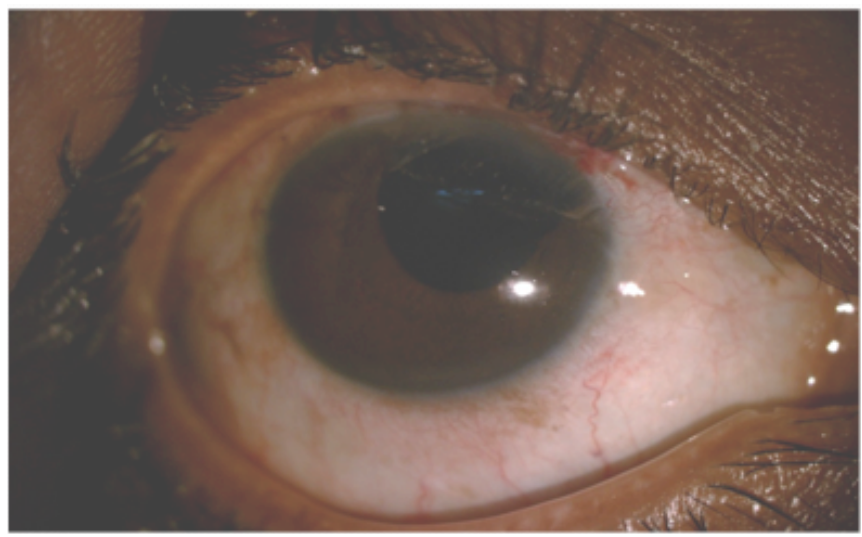

Fig. (3). Post-operative slit lamp photograph of the right eye showing a quiet eye with iridectomy and a posterior chamber intraocular lens implant.

There have been many procedures described in literature for the management of iris cysts with varying outcomes. Traditionally described treatment modalities for iris cysts involved extensive surgical procedures aimed at aggressive surgical excision and devitalization of the epithelial tissues. These included procedures like complete block excision of all epithelial layers and adjacent cornea, iris, anterior chamber angle and ciliary body, with full-thickness corneoscleral graft $[12,13]$, often combined with vitrectomy and lensectomy in phakic eyes, fluid-air exchange and cryodestruction of residual cells at the excision site $[6,14]$. However, these procedures, while generally successful in eradicating the epithelial tissue, involved extensive surgical procedures with the possibility of significant collateral damage to adjacent ocular structures, with resultant poor functional outcome [11]. More recently, many conservative surgical approaches to the management of iris cysts have been reported, including aspiration with or without cauterization, diathermy or iridectomy, injection of sclerosing agents, electrocautery and photocoagulation $[1,6$, $7,12]$. Viscoelastics have been used in the management of iris cysts to separate the cyst from the corneal endothelium so that the cyst may be excised with minimal trauma to the endothelium and adjacent ocular structures [15]. Xenon arc photoablation and argon laser photocoagulation of cysts have also been described [16, 17]. However, procedures like aspiration of the cyst have been reported to have a high rate of recurrence [2]. Laser treatment with Nd-YAG laser to rupture the cyst has also been reported, but with a poor outcome, with violent post-operative inflammatory reaction and high recurrence rates $[18,19]$.

The challenge in the surgical management of an iris cyst is to identify the complete extent of the epithelial down growth, and to ensure the removal of the cyst in toto [5]. If the cyst is ruptured, or part of the cyst wall or associated epithelial downgrowth is left behind, there is a high chance of recurrence. This can also cause violent post-operative inflammatory reaction with ensuing complications. On the other hand, aggressive surgical procedures aimed at complete eradication of the proliferative epithelial tissue are 
likely to produce significant collateral damage to the adjacent ocular structures, which in turn, would result in a poor functional outcome.

Our patient had a total cataract with epithelial downgrowth and extensive peripheral anterior synechiae, associated with the iris cyst. There was also mild pain and circumcorneal congestion, suggesting minimal ocular inflammation. The cyst was large enough to partially obscure the visual axis. We believed that excision of the cyst in toto with the involved iris and epithelial downgrowth would be vital to achieve a good surgical outcome in this case. We therefore, decided to perform the surgery through a large limbal incision, and excised the iris cyst in toto, with excision of a one millimeter margin of surrounding iris tissue and the epithelial downgrowth, combined with extracapsular cataract surgery and intraocular lens implantation through a large limbal incision. The patient's post operative period was uneventful, visual outcome was good and there was no significant inflammatory reaction associated with the procedure.

\section{CONCLUSION}

In conclusion, the optimal management of post-traumatic iris cysts often poses a challenge for ophthalmologists. A variety of surgical techniques, ranging from minimally invasive laser procedures to extensive surgical procedures like block excision, have been tried with variable outcomes [12]. However, the basic principle in the surgical management of secondary iris cysts seems to be that, as far as possible, the proliferative epithelial tissue which constitutes the cyst wall, and the associated epithelial downgrowth, have to be completely excised, while keeping the collateral tissue damage at a minimum level. Surgical procedures similar to the one described in our case can, therefore, achieve excellent functional outcomes.

\section{CONFLICT OF INTEREST}

The authors confirm that this article content has no conflict of interest.

\section{ACKNOWLEDGEMENTS}

\section{REFERENCES}

[1] Maumenee AE, Shannon CR. Epithelial invasion of the anterior chamber. Am J Ophthalmol 1956; 41: 929-42.

[2] Shields JA, Shields CL, Lois N, Mercado G. Iris cysts in children: classification, incidence, and management. Br J Ophthalmol 1999; 83: 334-8.

[3] Rizzuti AB. Traumatic implantation cysts of the iris with special emphasis on surgical aspects. Am J Ophthalmol 1955; 39: 13-20.

[4] Moreno-Lpez M, Arruabarrena C, Regueras A, Noval S. Conservative surgical management of a post-traumatic iris cyst. Arch Soc Esp Opthalmol 2007; 82: 455-8.

[5] Rishi P, Rishi E, Biswas J, Nandi K. Clinical and histopathological features of posttraumatic iris cyst. Indian J Ophthalmol 2008; 56: 518-21.

[6] Bruner WE, Michels RG, Stark WJ, Maumenee AE. Management of epithelial cysts of the anterior chamber. Ophthalmol Surg 1981; 12: 279-85.

[7] Tsai JA, Arrindell EL, O'Day DM. Needle aspiration and endodiathermy treatment of epithelial inclusion cyst of the iris. Am J Ophthalmol 2001; 131: 263-65.

[8] Marigo F, Finger P, McCormick S. Anterior segment implanation cysts. Arch Ophthalmol 1998; 116: 1569-75.

[9] Sitchevska O, Payne B. Pearl cyst of the iris. Am J Ophthalmol 1951; 34: 833-40.

[10] Terry T, Chrisholm J, Schonberg J. Differential diagnosis of anterior chamber cysts with ultrasound biomicroscopy. Acta Ophthalmol Scand 2006; 84: 137-39.

[11] Haller JA, Stark WJ, Azab AMR, Thomsen RW, Gottsch JD. Surgical management of anterior chamber epithelial cyst. Am J Ophthalmol 2003; 135: 309-13.

[12] Naumann GO, Rummelt V. Block excision of cystic and diffuse epithelial in growth of anterior chamber. Arch Ophthalmol 1992; 110: 223-7.

[13] Forster RK. Corneoscleral block excision of postoperative anterior chamber cysts. Trans Am Ophthalmol Soc 1995; 93: 83-97.

[14] Stark WJ, Michels RG, Maumenee AE, et al. Surgical management of epithelial ingrowth. Am J Ophthalmol 1978; 85: 772-80.

[15] Al-Ghadeer H, Al-Towerki AE, Al-Rajhi A, Al-Awad A. Longterm follow-up and visual outcome after excision of a traumatic iris cyst by viscoelastic dissection. Int Ophthalmol 2011; 31: 529-31.

[16] Cleasby GW. Photocoagulation of iris-ciliary-body epithelial cysts. Trans Am Acad Ophthalmol Otolaryngol 1971; 75: 638-42.

[17] Scholz RT, Kelly JS. Argon laser photocoagulation treatment of iris cysts following penetrating keratoplasty. Arch Ophthalmol 1982; 100: 926-7.

[18] Gupta V, Rao A, Sinha A, Kumar N, Sihota R. Post-traumatic inclusion cysts of the iris: A long term prospective case series. Acta Ophthalmol Scand 2007; 85: 893-6.

[19] Xiao Y, Wang Y, Niu G, Li K. Transpupillary argon laser photocoagulation and $\mathrm{Nd}$ _ YAG laser cystotomy for peripheral iris pigment epithelium cyst. Am J Ophthalmol 2006; 142: 691-3.

Declared none. 